\title{
The stability of astrophysical jets
}

\author{
Philip E. Hardee ${ }^{1}$ \\ ${ }^{1}$ Department of Physics \& Astronomy, Gallalee Hall, \\ The University of Alabama, Tuscaloosa, AL 35487 USA \\ email: phardee@bama.ua.edu
}

\begin{abstract}
Jets are produced by young stellar objects (YSOs), by black hole binary star system "microquasars" ( $\boldsymbol{\mu}$ QSOs), by active galactic nuclei (AGN), are associated with neutron stars and pulsar wind nebulae (PWNe), and are thought responsible for the gamma-ray bursts (GRBs). An understanding of these outflows must include how they are launched and collimated into jets, and how they propagate to large distances. Jets be they Poynting flux and/or kinetic flux dominated are current driven (CD) and/or Kelvin-Helmholtz (KH) velocity shear driven unstable. Here I present some of the work that is leading to a better understanding of the properties required for the observed relative stability of astrophysical jets.
\end{abstract}

Keywords. Galaxies: jets, ISM: jets and outflows, (magnetohydrodynamics:) MHD, instabilities, relativity

\section{Introduction}

Jetted outflows are associated with YSOs, e.g., Reipurth \& Bally (2001), $\mu$ QSOs, e.g., Mirabel \& Rodríguez (1999), AGN, e.g., Urry \& Padovani (1995); Ferrari (1998); Meier, Koide \& Uchida (2001), neutron stars and PWNe, e.g., Crab nebula jet (Weisskopf et al. 2000) and are thought responsible for the GRBs, e.g., Zhang \& Mészáros (2004); Piran (2005); Mészáros (2006). Jets are subject to current driven instability (CDI) where jets are magnetically dominated, and when kinetically dominated subject to the KelvinHelmholtz instability (KHI) driven by velocity shear between the jet and the surrounding medium. Instability is predicted theoretically from the linearized magnetofluid equations or, when the electromagnetic fields exceed the rest mass energy, the force-free "Poyntingflux" approximation. Thus, in astrophysical systems one might expect stability conditions to provide tools for selection between launching and propagation configurations satisfying the MHD equations governing jet flow. Jet structures attributed to instability are observed in laboratory experiments, e.g., Hsu \& Bellan (2002), in numerical simulations, e.g., Hardee et al. (1997), and in astrophysical jets.

In YSO systems the development of KHI driven structures strongly depends on the effects of radiative cooling. In general, radiative cooling appropriate to the ISM leads to enhanced stability relative to a non-radiatively cooled adiabatic jet (Massaglia et al. 1992; Micono et al. 1998). Note that strong magnetic fields are predicted to reduce the effects of radiative cooling, e.g., Hardee \& Stone (1997); Hardee, Stone \& Rosen (1997). For a recent review of KHI in YSO jets see the article by Trussoni (2009). While radiative cooling is not thought to be important on the relativistic jets there are still some interesting similarities in morphology between YSO jets and AGN jets, e.g., compare the knots, twists, asymmetries and bow shock structures in HH 212 (Zinnecker et al. 1998) and HH 111 (Reipurth et al. 1997; Reipurth et al. 1999) with the knots and twists in 3C 273 (Jester et al. 2005; Uchiyama et al. 2006) and the twists and bow shock structures in Hercules A (Dreher \& Feigelson 1984). Thus, the following discussion restricted to nonradiative $\mathrm{KHI}$ and $\mathrm{CDI}$ work will also be relevant to the YSO jets. 
Twisted structures are observed in many AGN jets on sub-parsec, parsec and kiloparsec scales, e.g., 3C 120 (Gómez et al. 2001), 3C 273 (Lobanov \& Zensus 2001) and M 87 (Lobanov, Hardee, \& Eilek 2003). The propagation, radial structure and growth or damping of instability produced jet structures depends on whether the structure is magnetically (CDI) or kinetically (KHI) induced. Non-relativistic and relativistic simulations of magnetized jet formation and/or propagation have shown helical structures attributed to CDI, e.g., (Lery et al. (2000); Nakamura et al. (2001); Ouyed et al. (2003); Nakamura \& Meier (2004); Nakamura et al. (2007); Moll et al. (2008); Moll(2009); McKinney \& Blandford (2009); Carey \& Sovinec (2009). Non-relativistic and relativistic simulations of kinetically dominated jets have shown that helical structures may be attributed to KHI, e.g., Hardee et al. (1997); Hardee et al. (2001); Hardee \& Hughes (2003). Large scale helical twisting may also be triggered by precession of the jet ejection axis (Begelman et al. 1980). It is still not clear whether current driven, velocity shear driven or jet precession is responsible for the observed structures, or whether these different processes are responsible for the observed twisted structures at different spatial scales.

\section{Kelvin-Helmholtz instability}

The KHI properties of jets in the kinetically dominated regime have been reviewed in various astrophysical contexts, e.g., Birkinshaw (1991a); Ferrari (1998); Hardee (2004); Hardee (2006); Trussoni (2008); Trussoni (2009); Keppens et al. (2009). Extensive analyses have been performed for fluid jets, e.g., Ferrari et al. (1978); Hardee (1979); Birkinshaw (1984); Hardee (1987); Hardee (2000), considered the effects of a velocity shear layer (Birkinshaw 1991b), and rotation (Bodo et al. 1996). Additional investigations added magnetic fields parallel to the flow velocity, e.g., Ferrari et al. (1980); Ferrari et al. (1981); Ferrari \& Trussoni (1983); Ray (1981); Londrillo (1985); Trussoni et al. (1988); Hardee (2007), and considered the addition of toroidal magnetic fields, e.g., Cohn (1983); Feidler \& Jones (1984); Appl \& Camenzind (1992). Relativistic jet simulations have found growth rates, e.g., Perucho et al. (2004a); Perucho et al. (2004b); Mizuno et al. (2007), and jet structure, e.g., Hardee et al. (1998); Hardee et al. (2001); Agudo et al. (2001); Hardee \& Hughes (2003), predicted theoretically.

Perturbations to a cylindrical jet can be considered to consist of Fourier components of the form $f_{1}(r, \phi, z, t)=f_{1}(r) \exp [i(k z \pm n \phi-\omega t)]$ where flow is along the $z$-axis and $n$ is an integer azimuthal wavenumber. The normal modes involve pinch $(n=0)$, helical $(n=1)$, elliptical $(n=2)$, triangular $(n=3)$, etc. (Hardee 1979) jet distortions and $+n$ and $-n$ specify the sense of spatial twist. Pinch structures can be triggered by flow variation associated with the central engine or jet overpressure. Twisted structures and the sense of twist can be induced by precession or orbital motion of the jet base, by rotation of the jet fluid, or by the helicity of the magnetic field.

For a super-magnetosonic jet with uniform density, pressure and velocity (top hat profile), and separated by a sharp boundary from an equal pressure uniform external environment, each normal mode formally consists of a fundamental and an infinite number of body mode solutions with different radial structure (see Fig. 5 in Hardee (2006); Fig. 13 in Hardee et al. (1997); Fig. 7 in Hardee et al. (2001)). The helical and higher order fundamental modes produce high pressure helically twisted filaments near to the jet's surface. The accompanying body modes produce helically twisted high pressure filaments in the jet's interior. The maximum fundamental mode growth rate when compared to the accompanying maximum body mode growth rates is less for the pinch, comparable for helical, and greater for elliptical and higher order normal modes. Spatial growth lengths 
scale with the Lorentz factor, magnetosonic Mach number and jet radius. In the subsonic but super-Alfvénic limit the body modes are stable but the jet surface remains unstable.

On astrophysical jets we expect a velocity shear layer to develop between the jet core and the external medium. This shear layer significantly modifies the growth of wavelengths comparable to the shear layer thickness, e.g., Ferrari et al. (1982); Ray (1982); Birkinshaw 1991b; Urpin (2002). There can be enhanced instability at short shear layer resonant wavelengths but, in general, growth rates are reduced by the presence of velocity shear, e.g., Perucho et al. (2005); Perucho et al. (2007). Still the growth rates are significant and the development of distortions to the jet's surface promotes mixing of the external medium into the jet fluid with the potential for disrupting the jet. Interestingly the analytically predicted shear layer resonances, which result in the formation of shocks and heating of the shear layer in numerical simulations, serves to stabilize the layer during non-linear evolution. The importance of the shear-layer resonant modes relies not only on their dominance among solutions of the linearized problem, but also on the fact that those jets for which the resonant modes are most important, do not disrupt and are very stable during the nonlinear evolution. It is noted that the steadiness of jets developing the shear layer resonant modes makes them firm candidates to remain collimated through long distances. Hence results would point to high Lorentz factor, highly supersonic jets as forming the FR II type jets, whereas the FR I type jets would be found in the opposite limit of slower and smaller Mach number jets.

The slow growth of large scale helical and elliptical twisting is not suppressed by the development of a velocity shear layer (Birkinshaw 1991b) and ultimately large scale helical twisting and to a lesser extent elliptical distortion can lead to catastrophic mixing and disruption of collimated jet flow, see Rosen et al. (1999). However, jets can remain highly collimated and avoid disruption by mixing by a multitude of effects associated with jet expansion. In the absence of magnetic fields, spatial growth of KHI is slowed significantly by increase in the Lorentz factor, increase in the Mach number and increase in the jet radius as spatial growth lengths scale with the Lorentz factor, the Mach number and the jet radius. Jet expansion also serves to push destructive helical twisting to slower growing longer wavelengths (relative to the jet radius). Along expanding jets waves can be advected down the jet to where their wavelength is shorter than the local fastest growing "resonant" wavelength. In addition to growth rate reduction in this shorter wavelength regime, the helical twist saturates at an amplitude that does not lead to significant surface distortion and accompanying mixing ( $\mathrm{Xu}$ et al. 2000).

In the presence of magnetic fields with magnetic and thermal pressures not too far from equipartition, linear KHI is modified partly by replacing sound speeds and accompanying Mach numbers with magnetosonic speeds and accompanying Mach numbers but significant magnetic helicity can enhance KHI or CDI depending on field helicity and strength Baty (2005). Additionally, the non-linear development of KHI can be significantly modified. Equipartition or weaker magnetic fields aligned with the velocity in a shear layer have been shown to be capable of stabilizing a shear profile through non-linear saturation, e.g., Frank et al. (1996); Jones et al. (1997); Keppens \& Tóth (2000); Ryu et al. (2000). The development of mixing can also be slowed by a helically twisted equipartition magnetic field configuration (Rosen et al. 1999). The toroidal magnetic field component reduces development of the elliptical and higher order normal modes, and simulations performed by Baty \& Keppens (2002) indicate a mode-mode interaction between KHI and CDI that prevents the $\mathrm{KH}$ vorticies from fully developing. Mixing is considerably reduced and only minimal reduction in collimation occurs. While mixing can never be completely suppressed on the $\mathrm{KH}$ unstable jet, effects of mixing on jet propagation also will be minimized if the jet core is denser than the immediately surrounding medium and 
entrains only lighter material, e.g., a hot tenuous shear layer or the tenuous radio lobes associated with FR II type radio sources like Cygnus A.

Numerical simulations have been used to study the development of KH pinch structures driven by velocity fluctuation in parsec-scale jets (Agudo et al. 2001), and twisted structures on AGN jets have been used to estimate the physical conditions in jets and in their surroundings Perucho et al. (2009). While this technique requires knowing jet and pattern speeds, the approach has been applied to the superluminal motions and accelerations of components along curved trajectories in the 3C 345 jet (Hardee 1987, Steffen et al. 1995), the milliarcsec twisted structure of the 3C 120 jet (Hardee et al. 2005), the twisted emission threads in the 3C 273 jet (Perucho et al. 2006) and the M 87 jet (Hardee \& Eilek 2010), and the transverse jet structure in 0836+710 (Perucho \& Lobanov 2007). Comparison between the $3 \mathrm{C} 120$ results and M 87 results is particularly interesting as the derived conditions are very different. The 3C 120 results (Hardee et al. 2005) indicate modest jet acceleration and cooling expansion along the inner kpc jet, effects that would serve to explain the relative stability and $\sim 100 \mathrm{kpc}$ jet length. On the other hand, the M 87 results (Hardee \& Eilek 2010) indicate considerable jet deceleration and heating along the kpc jet, effects that would serve to explain the observed destabilization beyond a few kpc.

\section{Current driven instability}

It is thought that the relativistic jets produced from rotating bodies (neutron stars, black holes and accretion disks) are powered and collimated hydromagnetically, e.g., Blandford (2000). A toroidal magnetic field is wound up in such outflows and in the far zone becomes dominant because the poloidal field falls off faster with expansion and distance. In configurations with strong toroidal magnetic field, the CD kink mode is unstable. This instability excites large-scale helical motions that can strongly distort or even disrupt the system. For static cylindrical force-free equilibria, the Kruskal-Shafranov criterion for instability, $\left|B_{\phi} / B_{p}\right|>2 \pi R / z$, indicates that the instability develops if the length of the plasma column is long enough for the field lines to go around the cylinder at least once, e.g., Bateman (1978). Non-relativistic theoretical work (Appl et al. 2000; Baty 2005) and numerical simulations (Lery et al. 2000; Baty \& Keppens 2002) show that CDI growth rates exceed KHI growth rates for sufficient magnetic field strength and helicity. CDI modes become dominant at increased helicity as the instability results from an imbalance between destabilizing toroidal and stabilizing poloidal magnetic field.

Poynting flux dominated outflows arise on magnetic field lines threading the horizon of a rotating black hole, e.g., Blandford \& Znajek (1977); Blandford \& Payne (1982). Jet acceleration and collimation is generally attributed to the toroidal magnetic field acting in concert with an external confining medium. For relativistic jet flow $\gamma_{j}>>1$ implies $E_{\text {kinetic }}>>W_{j} c^{2} \equiv\left\{\rho_{j}+[\Gamma /(\Gamma-1)] P_{j} / c^{2}\right\} c^{2}$. Here $\gamma_{j}$ is the jet Lorentz factor, $\Gamma$ is the adiabatic index, and $W$ is the enthalpy. For a magnetically produced jet we expect the jet speed $v_{j} \sim v_{A l f v e ́ n}$ and $\gamma_{j} \sim \gamma_{A} \sim\left[V_{A} / c\right] \equiv\left[B^{2} /\left(4 \pi W_{j} c^{2}\right)\right]^{1 / 2}>>1$ so there will be relatively low mass loading of the magnetic field lines. Here $B$ is the magnetic field measured in the jet rest frame and the jet is Poynting-flux dominated. In this regime the sound speed is less than the Alfvén wave speed, i.e., $a_{s}^{2} \equiv \Gamma P_{j} / W_{j}<v_{A}^{2} \equiv V_{A}^{2} /\left(1+V_{A}^{2} / c^{2}\right)$. A magnetically dominated jet with a uniform poloidal field component is $\mathrm{KH}$ stable when $\gamma_{j}<\gamma_{A}\left[1+\left(W_{j} / W_{e}\right)\left(V_{A, p}^{2} / c^{2}\right)\right]^{1 / 2}$ where $V_{A, p} \equiv\left[B_{p}^{2} /\left(4 \pi W_{j}\right)\right]^{1 / 2}$ and $W_{e}$ is the enthalpy of an unmagnetized external medium. Additional stabilization is provided by the presence of a significantly magnetized sheath or wind even if the jet core is super-Alfvénic (Hardee 
2007; Mizuno et al. 2007). The addition of a toroidal field component is not likely to alter this stability condition so we expect the Poynting-flux jet to be $\mathrm{KH}$ stable.

For magnetically dominated flows the magnetic field is likely to approximate a forcefree configuration. For relativistic force-free static configurations, analytical analyses of CDI criteria typically have been performed in the force-free Poynting-flux limit. The CDI kink mode, analogous to the KHI helical mode, is the most dangerous instability in configurations with toroidal magnetic field. This instability excites large scale helical motions that may disrupt the system. Cylindrical force-free jets are kink stable if the poloidal field is independent of the radius (Istomin \& Pariev 1994, Istomin \& Pariev 1996), but cylindrical force-free jets are kink unstable if the poloidal field decreases with the radius (Begelman 1998; Lyubarskii 1999). In the case of non-relativistic rotation, force-free jets are kink unstable if $\left|B_{\phi} / B_{p}\right|>|\Omega| R / c$ (Tomimatsu et al. 2001). All of these analyses are performed in a static reference frame, either infinite in extent or with a jet confined by rigid walls (Narayan et al. 2009). Provided the instability grows well inside the velocity shear surface defining a jet one might expect a CDI kink to move with $\gamma_{k i n k} v_{k i n k} \approx \gamma_{j} v_{j}$ and with "temporal" growth rate $\omega_{i} \propto\left(\gamma_{k i n k} R\right)^{-1}$ (Lyubarskii 1999). As a result we expect growth lengths $\ell_{e} \gtrsim \gamma_{j} R$ and for a typical jet with opening angle $\theta \sim f e w / \gamma_{j}$ the growth of the kink mode is relatively slow (Narayan et al. 2009). In a more realistic case, fluid inertia, relativistic rotation and velocity shear could significantly affect the instability criteria and the spatial growth of CDI. Unfortunately, no general stability analysis has been performed for magnetically dominated jets. Nevertheless, near to the central engine it is anticipated that CDI is the dominant instability.

The effects of magnetic helicity, jet density and jet flow profiles can be explored via numerical simulations. For static relativistic configurations (or more generally rigidly moving flows considered in the proper reference frame) with magnetic energy density comparable to or greater than the plasma energy density, Mizuno et al. (2009) found that the kink develops as predicted by linear theory but does not disrupt the initial force-free helical magnetic field. Growth rates are reduced by reduction in the Alfvén speed. Growth and morphological differences in the non-linear limit include a continually growing slender helically twisted column wrapped by magnetic field for magnetic helicity decreasing with radius, but growth nearly ceases for magnetic helicity increasing with radius. In all cases the characteristic time for the instability to affect strongly the initial structure for constant magnetic helicity is roughly $\tau \sim 100\left(a / v_{A}\right)$, where $a / v_{A}$ is the Alfvén crossing time across the characteristic radius, $a$, of the force-free magnetic field.

Mizuno et al. (2010) find that the introduction of a sub-Alfvénic weakly relativistic velocity shear surface has profound consequences for kink growth, propagation and the associated flow field. For the velocity shear surface well inside the characteristic radius, transverse growth is similar to the static case and the plasma flows through a growing non-moving kink. For the velocity shear surface well outside the characteristic radius, initial growth is similar to that of a static case and the kink is advected with the flow until the growing kink approaches the velocity shear surface. For velocity shear radii on the order of the characteristic radius there is a more intimate interaction between the growing kink and the flow field. In general, growth is slowed, the kink propagates more slowly than the flow and slows further as growth continues. Thus, there is some flow through the moving kink and more so as the kink grows and slows.

For constant magnetic pitch and declining density profile the characteristic time is roughly $\tau \sim 10 \tau_{e}$, with values for $\tau_{e}$ being dependent on the structure of the undisturbed state. In a jet context our perturbations remain static or can move with the flow frame depending on the location, $R_{j}$, of the velocity shear surface. In order to check whether the instability would affect a jet flow, one has to compare $\tau$ with a propagation time and 
present results suggest a scaling like $\tau \sim 10 \tau_{e} \equiv 10 \gamma_{k}^{\alpha} \tau_{e}^{s t}$ with $3 \geqslant \alpha \geqslant 1$ for a moving kink with $R_{j} \gtrsim a$. In this case the condition for the instability to affect the jet structure might be written as $z>A \gamma_{k}^{\alpha}\left(v_{k} / c\right) a$, where we set $10 \tau_{e}^{s t} \equiv A(a / c)$ and $0<v_{k} \leqslant v_{j}$ is a function of $R_{j} / a$ and is sensitively dependent on the location of the velocity shear surface provided $R_{j} / a<<10$. This result suggests that the characteristic spatial scale for kink development could be very much shorter or longer than for a kink simply advected with $v_{k}=v_{j} \sim c$ for which $z \gtrsim 100 \gamma_{j} a$. Non-relativistic analytic and numerical studies (Carey \& Sovinec 2009) indicate that jet rotation can provide a stabilizing influence.

The 3D relativistic jet generation simulation performed by McKinney \& Blandford (2009) indicates relatively rapid, less than 25 gravitational radii, but non-disruptive kink development over 500 gravitational radii. Our simulations for static and moving kinks suggest that the non-disruptive kink development in the jet generation simulation could be a result of the velocity shear surface located less or on order of the characteristic magnetic radius and a transverse density increase. This combination results in a slowly moving kink and rapid initial spatial development. Non-linear growth would be slowed by the transverse density increase, accompanying transverse Alfvén speed decline and increased Alfvén crossing time, associated with the confining denser slowly moving sheath found in jet generation simulations. Non-linear growth would also be significantly slowed by the jet expansion seen in jet generation simulations. Of course, a proper investigation of spatial growth requires stability simulations designed to study spatial kink development using realistic profiles.

It has been proposed that CDI converts Poynting flux to plasma energy flux and leads to a transition to kinetic flux jets. VLBA observations of the M87 jet (Walker et al. 2008, Walker et al. 2009) with a resolution of $0.21 \times 0.43$ mas provide an observational opportunity to constrain the Poynting flux region. The luminosity distance of M 87 is $\mathrm{D}=16.7 \mathrm{Mpc}$ (Mei et al. 2007) and the mass of the central black hole is $(6.0 \pm 0.5) \times$ $10^{9} M_{\odot}$ (Gebhardi \& Thomas 2009), at this distance. The Schwarzschild radius $R_{s} \sim$ $1.8 \times 10^{15} \mathrm{~cm}$ and 0.5 mas corresponds to $\sim 70 R_{s}$ in the sky plane. VLBA resolution is $\approx 230 R_{s}$ along the jet at a viewing angle of $15^{\circ}$ consistent with the $\sim 6 \mathrm{c}$ (Biretta et al. 1999) superluminal motions at a few hundred parsecs (HST-1). The VLBA structure is consistent with radio and optical kinetically dominated twisted structure at 10 parsec to kiloparsec scales (Hardee \& Eilek 2010). This suggests Poynting flux to plasma energy flux conversion within a few hundred gravitational radii of the black hole and places severe requirements on the jet structure that would allow CDI driven magnetic reconnection and reconfiguration on these spatial scales.

\section{Conclusion}

On relativistic jets CDI and KHI can produce twisted global structures mixed with structure induced by non-uniformity of the flow. In AGN Poynting flux to plasma energy flux conversion is likely restricted to the acceleration and collimation region at scales less than a few hundred gravitational radii from the black hole. The role of CDI in Poynting flux to plasma energy flux conversion is not yet determined but will depend on: (1) the global and/or sub-structure of the magnetic field in the acceleration region, (2) the gradients associated with jet acceleration and expansion, (3) the Lorentz factor of the flow, and (4) the global spine/sheath structure.

For jets to propagate to large distances and avoid disruptive mixing instigated by KHI they employ a variety of strategies to reduce entrainment. Stabilizing influences that reduce entrainment include: (1) strong or suitably ordered weaker magnetic fields, (2) suitable transverse gradients, (3) gradients such as those associated with jet acceleration 
or expansion, (4) high jet density (relative to the cocooning medium), and (5) high Lorentz factor and/or high Mach number.

\section{Acknowledgements}

P. Hardee acknowledges support from NASA (NNX08AG83G) and NSF (AST-0908010).

\section{References}

Agudo, I. et al. 2001, ApJL, 549, L183

Appl, S. \& Camenzind, M. 1992, A\& $A, 256,354$

Appl, S., Lery, T., \& Baty, H. 2000, A\&A A, 355, 818

Bateman, G. 1978, MHD Instabilites, (Cambridge, Mass., MIT Press), p.270

Baty, H. 2005, A\&A A, 430, 9

Baty, H. \& Keppens, R. 2002, ApJ, 580, 800

Begelman, M. C. 1998, ApJ, 493, 291

Begelman, M. C., Blandford, R. D. \& Rees, M. J. 1980, Nature, 287, 307

Biretta, J. A., Sparks, W. B., \& Macchetto, F. 1999, ApJ, 520, 621

Birkinshaw, M. 1984, MNRAS, 208, 887

Birkinshaw, M. 1991, Beams and Jets in Astrophysics, ed. P. Hughes, p.278

Birkinshaw, M. 1991, MNRAS, 252, 73

Blandford, R. D. 2000, Phil.Trans.Roy.Soc. A, 358, 1

Blandford, R. D. \& Payne, D. G. 1982, MNRAS, 199, 883

Blandford, R D. \& Znajek, R. L. 1977, MNRAS, 179, 433

Bodo, G., Rosner, R., Ferrari, A., \& Knoblock, E. 1996, ApJ, 470, 797.

Carey, C. S., \& Sovinec, C. R. 2009, ApJ, 699, 362

Cohn, H. 1983, ApJ, 269, 500

Dreher, J., \& Feigelson, E. 1984, Nature, 308, 43

Feidler, R. \& Jones, T. W. 1984, ApJ, 283, 532

Ferrari, A. 1998, ARA\& $\&$, 36, 539

Ferrari, A., Massaglia, S., \& Trussoni, E. 1982, MNRAS, 198, 106

Ferrari, A. \& Trussoni, E. 1983, MNRAS, 125, 179

Ferrari, A., Trussoni, E., \& Zaninetti, L. 1978, A\&A, 64, 43

Ferrari, A., Trussoni, E., \& Zaninetti, L. 1980, MNRAS, 193, 469

Ferrari, A., Trussoni, E., \& Zaninetti, L. 1981, MNRAS, 196, 105

Frank, A., Jones, T. W., Ryu, D., \& Gaalaas, J. B. 1996, ApJ, 460, 777

Gebhardt, K. \& Thomas, J. 2009, ApJ, 700, 1690

Gómez, J. L. et al. 2001, ApJ, 561, L161

Hardee, P. E. 1979, ApJ, 234, 47

Hardee, P. E. 1987, ApJ, 318, 78

Hardee, P. E. 2000, ApJ, 533, 176

Hardee, P. E. 2004, ApSS, 293, 117

Hardee, P. E. 2006, AIP Conf. Series 856, eds. Hughes \& Bregman, p.57

Hardee, P. E. 2007, ApJ, 664, 26

Hardee, P. E., Clarke, D. A., \& Rosen, A. 1997, ApJ, 485, 533

Hardee, P. \& Eilek, J. 2010, in preparation

Hardee, P. E. \& Hughes, P. A. 2003, ApJ, 583, 116.

Hardee, P. E., Hughes, P. A., Rosen, A., \& Gomez, E. A. 2001, ApJ, 555, 744.

Hardee, P. E., Rosen, A., Hughes, P. A., \& Duncan, G. C. 1998, ApJ, 500, 599

Hardee, P. E., Walker, R. C., \& Gómez, J. L. 2005, ApJ, 620, 646

Hardee, P. E. \& Stone, J. M., 1997, ApJ, 483, pp.121-135 (1997)

Hardee, P. E., Stone, J. M., \& Rosen, A., 1997, IAU Symp. 182, eds. Malbet \& Castets, p.132

Hsu, S. C. \& Bellan, P. M. 2002, MNRAS, 334, 257

Istomin, Y. N. \& Pariev, V. I. 1994, MNRAS, 267, 629 
Istomin, Y. N. \& Pariev, V. I. 1996, MNRAS, 281, 1

Jester, S., Röser, H.-J., Meisenheimer, K., \& Perley, R. 2005, A\&A, 431, 447

Jones, T. W., Gaalaas, J. B., Ryu, D., \& Frank, A. 1997, ApJ, 482, 230

Keppens, R. \& Tóth, G. 2000, Phys.Plasmas, 6, 1461

Keppens, R., Meliani, Z., Baty, H., \& van der Holst, B. 2009, Lect. Notes Phys., 791, 179

Lery, T., Baty, H., \& Appl, S. 2000, A\&A, 355, 1201

Lobanov, A., Hardee, P., \& Eilek, J. 2003, New Science Reviews, 47, 629

Lobanov, A. P. \& Zensus, J. A. 2001, Science, 294, 128

Londrillo, P. 1985, A\&BA, 145, 353

Lyubarskii, Y. E. 1999, MNRAS, 308, 1006

Massaglia, S., Trussoni, E., Bodo, G., Rossi, P., \& Ferrari, A. 1992, A\&A A, 260, 243

McKinney, J. C. \& Blandford, R. D., 2009, MNRAS, 394, L126

Mei et al. 2007, ApJ, 655, 144

Mészáros, P. 2006, Rep.Prog.Phys., 69, 2259

Micono, M., Bodo, G., Massaglia, S., Rossi, P., \& Ferrari, A. 1992, A\&A, 260, 243

Meier, D. L., Koide, S., \& Uchida, Y. 2001, Science, 291, 84

Mirabel, I F. \& Rodríguez, L. F. 1999, ARA\&A, 37, 409

Mizuno, Y., Hardee, P., \& Nishikawa, K-I. 2007, ApJ, 662, 835

Mizuno, Y., Lyubarsky, Y., Nishikawa, K.-I., \& Hardee, P. E. 2009, ApJ, 700, 684

Mizuno, Y., Hardee, P., \& Nishikawa, K.-I. 2010, in preparation

Moll, R. 2009, A\&A, 507, 1203

Moll, R., Spruit, H. C., \& Obergaulinger, M. 2008, A\& $A, 492,621$

Nakamura, M., Uchida, Y., \& Hirose, S. 2001, New Astronomy, vol.6, issue 2, 61

Nakamura, M., \& Meier, D. L. 2004, ApJ, 617, 123

Nakamura, M., Li, H., \& Li, S. 2007, ApJ, 656, 721

Narayan, R., Li, J., \& Tchekhovskoy, A. 2009, ApJ, 697, 1681

Ouyed, R., Clarke, D. A., \& Pudritz, R. E. 2003, ApJ, 582, 292

Perucho, M., Hanasz, M., Martí, J. M., \& Sol, H. 2004a, A 6 A, 427, 415

Perucho, M., Hanasz, M., Martí, J. M., \& Miralles, J. A. 2007, Phys.Rev.E, 75, 631

Perucho, M., Lobanov, A. P., \& Kovalev, Y. Y. 2009, ASPCS, 402, 349

Perucho, M. \& Lobanov, A. P. 2007, A\&A, 469, 23

Perucho, M., Lobanov, A. P., Martí, J.-M., \& Hardee, P. E. 2006, A\&A, 456 , 493

Perucho, M., Martí, J. M., \& Hanasz, M. 2004b, A\&SA, 427, 431

Perucho, M., Martí, J. M., \& Hanasz, M. 2005, A\&A A, 443, 863

Piran, T. 2005, Reviews of Modern Physics, 76, 1143

Ray, T. P. 1981, MNRAS, 196, 195

Ray, T. P. 1982, MNRAS, 198, 617

Reipurth, B. \& Bally, J. 2001, ARA\&A, 39, 403

Reipurth, B., Hartigan, P., Heathcote, S., Morse, J., \& Bally, J. 1997, AJ, 114, 757

Reipurth, B., Yu, K., Rodrguez, L., Heathcote, S., \& Bally, J. 1999, A\& $A$, 352, 83

Rosen, A., Hardee, P. E., Clarke, D. A., \& Johnson, A. 1999, ApJ, 510, 136.

Ryu, D., Jones, T. W., \& Frank, A. 2000, ApJ, 545, 475.

Steffen, W., Zensus, J. A., Krichbaum, T. P., Witzel, A., \& Qian, S. J. 1995, A\& A, 302, 335

Tomimatsu, A., Matsuoka, T., \& Takahashi, M. 2001, Phys.Rev.D, 64, 123003

Trussoni, E., Massaglia, S., Bodo, G., \& Ferrari, A. 1988, MNRAS, 234, 539

Trussoni, E. 2008, Jets from Young Stars III - Numerical MHD and Instabilities, eds. Massaglia et al. LNP 754 (Springer: Berlin Heidelberg), p.105

Trussoni, E. 2009, Protostellar Jets in Context, eds. Tsinganos, Ray \& Stute (Springer: Berlin Heidelberg), p.285

Uchiyama, Y. et al. 2006, ApJ, 648, 910

Urpin, V. 2002, A\&A A, 385, 14

Urry, C. M. \& Padovani, P. 1995, PASP, 107, 903

Walker, R. et al. 2008, JPhCS, 131, 012053

Walker, R. et al. 2009, ASPCS, 402, eds. Hagiwara et al., (San Francisco, ASP), p. 227 
Weisskopf, C. et al. 2000, ApJ, 536, L81

$\mathrm{Xu}$, J., Hardee, P., \& Stone, J. 2000, ApJ, 543, 161

Zhang, B. \& Mészáros, P. 2004, Int.J.Mod.Phys., A19, 2385

Zinnecker, H., McCaughrean, M., \& Rayner, J. 1998, Nature, 394, 862

\section{Discussion}

SIKORA: What determines the polarity of twisted modes triggered by KH instabilities?

HARDEE: The polarity of twisted modes is determined by the rotation and associated heliciy of the flow and magnetic fields. 